\title{
A REMARK ON THE MODULI OF RIEMANN SURFACES OF GENUS 2
}

\section{P. R. GARABEDIAN ${ }^{1}$}

1. Introduction. The object of this paper is to show how the problem of determining moduli of conformal type for certain Riemann surfaces can be solved by using the theory of Riemann theta-functions. It is to be hoped that the basic ideas presented here, together with the results of Schottky [6] ${ }^{2}$ and Poincare [5] on relations between periods of integrals of the first kind on closed Riemann surfaces, will lead to further work with the theta-function method on the problem of determining conformal moduli.

Our principal theorem is an extension to closed surfaces of genus 2 of work done by Ahlfors and Beurling [1] on the moduli of triplyconnected regions. Their result is found to be a special case of the theorem for closed surfaces, as will be seen from a consideration of the double of the triply-connected region, which is a symmetric closed surface of genus 2. The method of theta-functions used in this paper is, of course, quite different from the method of extremal length developed by Ahlfors and Beurling. Relations between the two methods and effective extensions of both to the treatment of moduli of more general Riemann surfaces offer an interesting field for further study. We remark that results on infinitesimally close Riemann surfaces have been obtained already in the general case by use of variational methods [2].

2. Riemann theta-functions. We consider a closed Riemann surface $S$ of genus 2 . On $S$ we introduce the usual system of canonical cuts $\alpha_{1}, \alpha_{2}, \beta_{1}, \beta_{2}$, and we define two linearly independent normal integrals of the first kind, $w_{1}(z)$ and $w_{2}(z)$, for $z$ in $S$. The integral $w_{\mu}(z)$ has about $\alpha_{1}$ and $\alpha_{2}$ periods $\delta_{\mu 1} \pi i$ and $\delta_{\mu 2} \pi i$, and has about $\beta_{1}$ and $\beta_{2}$ periods $a_{\mu 1}$ and $a_{\mu 2}, \mu=1,2$, where $\delta_{\mu \nu}$ is the Kronecker delta. The quadratic form

$$
\sum_{\mu, \nu=1}^{2} \operatorname{Re}\left\{a_{\mu \nu}\right\} x_{\mu} x_{\nu}
$$

is negative definite, when $x_{1}$ and $x_{2}$ are real. Also, we have the symmetry relation $a_{\mu \nu}=a_{\nu \mu}$.

Presented to the Society, October 29, 1949; received by the editors July 25, 1949.

1 National Research Fellow.

2 Numbers in brackets refer to the bibliography at the end of the paper. 
We define the Riemann theta-function

$$
\theta\left(u_{1}, u_{2}\right)=\sum_{n_{1}, n_{2}=-\infty}^{\infty} \exp \left\{\sum_{\mu, v=1}^{2} a_{\mu} n_{\mu} n_{\nu}+2 \sum_{\mu=1}^{2} u_{\mu} n_{\mu}\right\}
$$

associated with the closed Riemann surface $S$. This series converges for all complex values of $u_{1}, u_{2}$, and it represents an analytic function of considerable importance for the consideration of functions on $S$. Riemann found, using the theory of this theta-function, that every pair of complex numbers $V_{1}, V_{2}$ has a representation

$$
V_{1}=w_{1}\left(z_{1}\right)+w_{1}\left(z_{2}\right), \quad V_{2}=w_{2}\left(z_{1}\right)+w_{2}\left(z_{2}\right),
$$

where $z_{1}$ and $z_{2}$ are points on $S$, and he showed that this representation is in general unique (Jacobi inversion problem). Furthermore, he proved the following representation, which is of fundamental significance for our work and is connected with the uniqueness of the representation (2). If the additive constants implicitly contained in the definition of the integrals of the first kind $w_{1}(z)$ and $w_{2}(z)$ on $S$ are suitably chosen, then the zeros $V_{1}, V_{2}$ of the function $\theta\left(u_{1}, u_{2}\right)$, that is, the points $V_{1}, V_{2}$ with

$$
\theta\left(V_{1}, V_{2}\right)=0,
$$

are given by

$$
V_{1}=w_{1}(z), \quad V_{2}=w_{2}(z),
$$

where $z$ is a variable point of $S$, and this representation is in general unique. We shall consider throughout this paper that the constants implicitly contained in the integrals $w_{1}(z)$ and $w_{2}(z)$ have been chosen so that (3) holds.

A detailed presentation of the notions and results which we have described here is given by Neumann [4]. We remark that an inversion formula of the form (2) completely solves the problem of finding moduli for the closed Riemann surfaces of genus 1 . Indeed, such a formula leads to the complete discussion of these surfaces in terms of elliptic functions. Our principal object now will be to use the more refined formula (3) in order to find moduli for the closed Riemann surfaces $S$ of genus 2 .

3. Moduli of conformal type. The Riemann surface $S$ which we have discussed depends in its conformal type upon 6 real parameters, which we call a set of conformal moduli of $S$. That is, with each such surface we can associate an ordered set of 6 real numbers in such a way that there is a conformal mapping of preassigned topological 
character of any one such surface, $S$, upon any other, $S^{*}$, if and only if the corresponding sets of 6 numbers are identical. We shall prove in this section that the real and imaginary parts of the three complex numbers $a_{11}, a_{12}$, and $a_{22}$ yield a set of conformal moduli of $S$ of the kind we describe. By virtue of the identity $a_{12}=a_{21}$, we see that this will be proved if we show that the matrix $\left(\left(a_{\mu \nu}\right)\right)$ determines the conformal type of $S$ uniquely.

Let $S^{*}$ be a second closed Riemann surface of genus 2 , and associate with $S^{*}$ a system of canonical cuts $\alpha_{1}^{*}, \alpha_{2}{ }^{*}, \beta_{1}{ }^{*}, \beta_{2}{ }^{*}$ and a corresponding pair of normalized integrals of the first kind $w_{1}^{*}\left(z^{*}\right)$, $w_{2}^{*}\left(z^{*}\right)$. Also to be associated with $S^{*}$ is a matrix of periods $\left(\left(a_{\mu \nu}^{*}\right)\right)$ of the integrals $w_{1}^{*}\left(z^{*}\right)$ and $w_{2}^{*}\left(z^{*}\right)$ and a Riemann theta-function $\theta^{*}\left(u_{1}, u_{2}\right)$. We shall show that if the matrices $\left(\left(a_{\mu \nu}\right)\right)$ and $\left(\left(a_{\mu \nu}^{*}\right)\right)$ are identical, that is, if $a_{11}=a_{11}^{*}, a_{12}=a_{12}^{*}$, and $a_{22}=a_{22}^{*}$, then $S$ can be mapped conformally and one-one upon $S^{*}$, and the mapping which we shall construct in the proof will send the set of canonical cuts $\alpha_{1}, \alpha_{2}, \beta_{1}, \beta_{2}$ into a set of canonical cuts on $S^{*}$ which are equivalent topologically to $\alpha_{1}^{*}, \alpha_{2}^{*}, \beta_{1}^{*}, \beta_{2}^{*}$.

Indeed, suppose that $\left(\left(a_{\mu \nu}\right)\right)$ and $\left(\left(a_{\mu \nu}^{*}\right)\right)$ are identical. Then from the definition (1) of the theta-function $\theta\left(u_{1}, u_{2}\right)$ and the corresponding definition of $\theta^{*}\left(u_{1}, u_{2}\right)$, we obtain the identity

$$
\theta\left(u_{1}, u_{2}\right) \equiv \theta^{*}\left(u_{1}, u_{2}\right) \text {. }
$$

Hence the manifold of zeros $V_{1}, V_{2}$ of $\theta\left(u_{1}, u_{2}\right)$ is the same as the manifold of zeros $V_{1}, V_{2}$ of $\theta^{*}\left(u_{1}, u_{2}\right)$. Thus we have not only the representation (3) of each point $V_{1}, V_{2}$ on this manifold, but also the representation

$$
V_{1}=w_{1}^{*}\left(z^{*}\right), \quad V_{2}=w_{2}^{*}\left(z^{*}\right),
$$

where $z^{*}$ is a variable point on $S^{*}$. Thus we obtain the relations

$$
w_{1}(z)=w_{1}^{*}\left(z^{*}\right), \quad w_{2}(z)=w_{2}^{*}\left(z^{*}\right),
$$

between suitable points $z$ on $S$ and $z^{*}$ and $S^{*}$.

We maintain that the correspondence (6), which is in general oneone, yields a conformal mapping $z^{*}(z)$ of $S$ upon $S^{*}$ and at the same time yields the inverse mapping $z\left(z^{*}\right)$ of $S^{*}$ upon $S$. These mappings can, indeed, be obtained by solving the first implicit equation (6) first for $z^{*}$ and then for $z$. The solutions can break down only at the critical points of $w_{1}^{*}\left(z^{*}\right)$ on $S^{*}$ and the critical points of $w_{1}(z)$ on $S$. But near these points, we can use the second implicit equation (6) in order to determine the mappings $z^{*}(z)$ and $z\left(z^{*}\right)$. Indeed, the integrals $w_{1}(z)$ and $w_{2}(z)$ cannot have a critical point in common, nor can 
the integrals $w_{1}^{*}\left(z^{*}\right)$ and $w_{2}^{*}\left(z^{*}\right)$.

To prove the last statement, say for the pair of integrals $w_{1}(z)$ and $w_{2}(z)$, we remark that by the Riemann-Roch theorem the functions $w_{1}(z)$ and $w_{2}(z)$ each have precisely two critical points on $S$. Thus if $w_{1}(z)$ and $w_{2}(z)$ had a critical point in common, then the single-valued analytic function

$$
\frac{d w_{1}(z)}{d w_{2}(z)}
$$

would have either one pole or no poles on $S$, and hence it would be either univalent or constant on $S$. But both these possibilities are excluded by the fact that $S$ has genus 2 , and thus we should arrive at a contradiction.

Coming back once more to the equations (6), we see that their solutions $z^{*}(z)$ and $z\left(z^{*}\right)$ on $S$ and $S^{*}$ are single-valued functions, with values in $S^{*}$ and $S$, since the period matrices $\left(\left(a_{\mu \nu}\right)\right)$ and $\left(\left(a_{\mu \nu}^{*}\right)\right)$ are identical. Thus, indeed, we obtain a one-one conformal mapping of $S$ upon $S^{*}$, as desired. It follows that the matrix $\left(\left(a_{\mu \nu}\right)\right)$ yields a set of 6 real moduli for $S$, as stated above. ${ }^{3}$

4. Symmetric cases. The result of the previous section can be used to obtain a theorem of the type investigated by Ahlfors and Beurling [1] for triply-connected regions. Let $D$ be a domain of the complex $z$-plane bounded by three curves $C_{1}, C_{2}$, and $C_{3}$, which we may assume with no loss of generality to be analytic. Let $\omega_{\nu}(z)$ be that harmonic function in $D$ with boundary values 1 on $C_{\nu}$ and zero boundary values on the $C_{\mu}$ with $\mu \neq \nu, \nu=1,2,3$. Let $W_{\nu}(z)$ be the corresponding analytic function in $D$ with $\omega_{\nu}(z)=\operatorname{Im}\left\{W_{\nu}(z)\right\}$. The three functions $W_{\nu}(z)$ have in $D$ a matrix of real periods, $\left(\left(p_{\mu \nu}\right)\right)$, and these periods are completely determined by the subset $p_{11}, p_{12}$, and $p_{22}$. We maintain that $p_{11}, p_{12}$, and $p_{22}$ are a set of three conformal moduli for the triply-connected domain $D$, and we shall prove this using the result of $\$ 3$, although simpler, elementary proofs are available.

We can associate with $D$ a closed Riemann surface $S$ of genus 2, known as the double of $D$. The surface $S$ is obtained by taking two replicas of $D$ and identifying corresponding boundary points. The

3 This procedure can also be used to show that there is always a nontrivial conformal mapping of $S$ onto itself. Indeed, since $\theta\left(u_{1}, u_{2}\right)$ is an odd function, we have in addition to (3) the representation $V_{1}=-w_{1}(z), V_{2}=-w_{2}(z)$ of the zeros of $\theta\left(u_{1}, u_{2}\right)$. Thus we can define a conformal mapping $\tilde{z}(z)$ of $S$ onto itself by means of the relations $w_{1}(\bar{z})=-w_{1}(z), w_{2}(\tilde{z})=-w_{2}(z)$. 
normal integrals of the first kind $w_{1}(z)$ and $w_{2}(z)$ on $S$ can be chosen so that

$$
w_{\mu}(z)=\frac{\pi}{2} W_{\mu}(z)+\text { const., } \quad \mu=1,2,
$$

in $D$. Thus the matrix $\left(\left(a_{\mu v}\right)\right)$ for $S$ is given by the relations

$$
2 a_{11}=\pi p_{11}, \quad 2 a_{12}=\pi p_{12}, \quad 2 a_{22}=\pi p_{22} .
$$

We see immediately that if the periods $p_{11}, p_{12}, p_{22}$ associated with the domain $D$ are identical with the periods $p_{11}^{*}, p_{12}^{*}, p_{22}^{*}$ associated with a second triply-connected plane domain $D^{*}$, then the double $S$ of $D$ can be mapped conformally upon the double $S^{*}$ of $D^{*}$, by $\S 3$.

Let $z^{*}(z)$ be the mapping of $S$ upon $S^{*}$. Then we have from (6) and (7), for a suitably chosen real constant $\lambda$, the relation

$$
\omega_{1}^{*}\left(z^{*}(z)\right)=\omega_{1}(z)+\lambda,
$$

where $\omega_{\mu}^{*}\left(z^{*}\right), \mu=1,2,3$, are the harmonic functions in $D^{*}$ corresponding to the $\omega_{\mu}(z)$ in $D$, with boundary values 1 or 0 on the respective boundary components of $D$. Now the critical points of $\omega_{1}^{*}\left(z^{*}\right)$ and $\omega_{1}(z)$ must correspond in the mapping $z^{*}(z)$, since this mapping is single-valued and univalent. Let $z_{0}$ and $z_{0}^{*}$ be a pair of corresponding critical points, and let $z_{1}$ and $z_{1}{ }^{*}$ be the points symmetric to $z_{0}$ and $z_{0}{ }^{*}$ on $S$ and $S^{*}$. Of course, $z_{1}$ and $z_{1}{ }^{*}$ are critical points of $w_{1}(z)$ and $w_{1}^{*}\left(z^{*}\right)$, respectively, by symmetry.

We can continue the identity (9) analytically from $z_{0}$ to $z_{1}$, across $C_{2}$. We have thus on the one hand

$$
\omega_{1}^{*}\left(z^{*}\left(z_{0}\right)\right)=\omega_{1}\left(z_{0}\right)+\lambda,
$$

while on the other hand, letting $z=z_{1}$ in (9), we obtain

$$
-\omega_{1}^{*}\left(z^{*}\left(z_{0}\right)\right)=-\omega_{1}\left(z_{0}\right)+\lambda,
$$

since $\omega_{1}\left(z_{1}\right)=-\omega_{1}\left(z_{0}\right), \omega_{1}^{*}\left(z_{1}^{*}\right)=-\omega_{1}^{*}\left(z_{0}^{*}\right)$. Adding (10) and (11), we have

$$
2 \lambda=0,
$$

and thus (9) takes the simpler form

$$
\omega_{1}^{*}\left(z^{*}(z)\right)=\omega_{1}(z) .
$$

It is clear from (12) that the conformal mapping $z^{*}(z)$ of $S$ upon $S^{*}$ yields, in particular, a conformal mapping of $D$ upon $D^{*}$. The desired theorem on the conformal moduli of triply-connected domains follows as an immediate consequence, since this proves that $p_{11}$, 
$p_{12}$, and $p_{22}$ are a set of conformal moduli for $D$.

We remark, finally, that in a similar way we can find a set of three conformal moduli for the Riemann surfaces $R$ of genus 1 with 1 boundary curve. Here again, the construction depends upon a consideration of the symmetric double $S$ of $R$, obtained by identifying boundary points of two replicas of $R$. Clearly, the double $S$ of $R$ has genus 2 , and the period matrix $\left(\left(a_{\mu \nu}\right)\right)$ associated with $S$ satisfies three independent symmetry relations, namely, $a_{11}=\bar{a}_{22}, a_{12}=\bar{a}_{21}$, so that we are left with 3 real conformal moduli for $R$. The detailed investigation of this second symmetric case is left to the reader, and its discussion will lead to a more complete understanding of the present method. Suffice it to say that here the role which the harmonic functions $\omega_{\mu}(z)$ played for $D$ is played by the integrals over the two canonical cuts on $R$ of the normal derivative of the Green's function of $R$.

\section{BIBLIOGRAPHY}

1. L. Ahlfors and A. Beurling, Invariants conformes et problèmes extrémaux II, Comptes Rendus du Dixième Congrès des Mathématiciens Scandinaves, Copenhagen, 1947, pp. 341-351.

2. P. R. Garabedian and M. Schiffer, Identities in the theory of conformal mapping, Trans. Amer. Math. Soc. vol. 65 (1949) pp. 187-238.

3. A. Krazer, Lehrbuch der Thetafunktionen, Leipzig, 1903.

4. C. Neumann, Vorlesungen über Riemanns Theorie der Abel'schen Integrale, Leipzig, 1884.

5. H. Poincaré, Remarques diverses sur les fonctions abéliennes, J. Math. Pures Appl. (5) vol. 1 (1895) pp. 219-314.

6. F. Schottky, Zur Theorie der Abelschen Functionen von vier Variabeln, J. Reine Angew. Math. vol. 102 (1888) pp. 304-352.

STANFORD UNIVERSITY 